\title{
Amplification of temporally modulated signal beams by two-wave mixing in $\mathrm{Bi}_{12} \mathrm{SiO}_{20}$
}

\author{
D. J. Webb and L. Solymar \\ Holography Group, Department of Engineering Science, Oxford University, Parks Road, Oxford, OX1 3PJ, UK
}

Received May 18, 1990; accepted July 24, 1990

\begin{abstract}
A theoretical analysis of two-wave mixing in a BSO crystal is developed in the undepleted-pump approximation for a modulated signal beam. It is shown that, for a modulation of high enough frequency, significant ac amplification is possible at three distinct values of pump-beam detuning. A signal beam that is amplitude modulated by a square wave is analyzed by means of the theory, and experimental results are presented in confirmation of the analysis. Finally, it is shown that in the presence of absorption the optimum detunings for dc and ac amplification are different.
\end{abstract}

\section{INTRODUCTION}

Two-wave mixing in photorefractive media has been studied theoretically and experimentally by a number of researchers, and a wide range of prospective applications of the effect have been demonstrated or proposed. One such application, described recently by Hamel de Montchenault and co-workers, concerns the amplification of high-frequency amplitude-modulated signals by two-wave mixing in $\left.\mathrm{BSO}\left(\mathrm{Bi}_{12} \mathrm{SiO}_{20}\right)\right)^{1,2}$ In this paper we present an analysis of two-wave mixing with modulated signal beams in the undepleted-pump-beam approximation and describe further experimental results that compare favorably with the theoretical predictions.

\section{THEORY}

We assume that incident on the photorefractive crystal are a strong pump beam of amplitude $P(0)$ and an amplitudemodulated signal beam of amplitude $S(0, t)$, the geometry being as indicated in Fig. 1 and the interbeam angle being small. Further, we allow for the pump beam to be detuned by an amount $\delta \omega$; detuning may be conveniently carried out with the aid of the piezoelectric mirror shown. The dependence of the amplitudes of the two beams and the space-charge field $E_{\mathrm{SC}}$ on the distance into the crystal, $z$, may be obtained from Kukhtarev's equations ${ }^{3,4}$ as

$$
\begin{aligned}
\frac{\partial E_{\mathrm{SC}}}{\partial t}+\frac{E_{\mathrm{SC}}}{\tau_{d}} & =\frac{E_{Q} B P^{*}(z) S(z, t)}{\tau_{d} I_{0}} e^{-j \delta \omega t}, \\
\frac{\partial S(z, t)}{\partial z} & =-j \frac{\Gamma E_{\mathrm{SC}} P(z)}{E_{Q}} e^{j \delta \omega t}-\alpha S(z, t), \\
\frac{\partial P(z)}{\partial z} & =-j \frac{\Gamma E_{\mathrm{SC}}{ }^{*} S(z, t)}{E_{Q}} e^{-j \delta \omega t}-\alpha P(z),
\end{aligned}
$$

where $E_{\mathrm{SC}}$ is the amplitude of the space-charge field, $I_{0}$ is the total power density, $\tau_{d}$ is the dielectric relaxation time, $\alpha$ is the amplitude absorption coefficient, and $A, B, E_{\mathrm{Q}}$, and $\Gamma$ depend on the material parameters, system geometry, and source wavelength but are here regarded as constants.
In the undepleted-pump-beam approximation, where the pump is assumed to be much stronger than the signal beam, $P$ is affected only by absorption within the material and hence varies as

$$
P(z)=P(0) e^{-\alpha z} .
$$

In applications involving the amplification of weak signals this is a reasonable approximation. Equations (1), (2), and (4) may then be combined to eliminate $E_{\mathrm{SC}}$, and the spatial and temporal variation of the signal beam is then determined by

$$
\begin{aligned}
\frac{\partial^{2} S(z, t)}{\partial z \partial t}+\alpha \frac{\partial S(z, t)}{\partial t} & +\left(\frac{A}{\tau_{d}}-j \delta \omega\right) \frac{\partial S(z, t)}{\partial z} \\
+ & {\left[\alpha\left(\frac{A}{\tau_{d}}-j \delta \omega\right)+j \frac{\Gamma B}{\tau_{d}}\right] S(z, t)=0 . }
\end{aligned}
$$

Here the intensity dependence of the dielectric relaxation time must be taken into account by letting $\tau_{d}=$ $\tau_{d 0}[\exp (2 \alpha z)]$, where $\tau_{d 0}$ is the dielectric relaxation time at the front of the crystal. ${ }^{5}$

Since we are interested in signals that are periodic in time, we shall look for a solution of the form

$$
S(z, t)=a_{1}(z) e^{j \Omega t},
$$

where $\Omega$ is an angular frequency and $a_{1}(0)$ is assumed to be given. The required solution may readily be obtained as

$$
a_{1}(z)=a_{1}(0)\left[\frac{A+j(\Omega-\delta \omega) \tau_{d 0}}{A e^{-2 a z}+j(\Omega-\delta \omega) \tau_{d 0}}\right]^{-j(\Gamma B / 2 \alpha A)} e^{-\alpha z} .
$$

Since any periodic modulation function may be expressed as a Fourier series,

$$
S(0, t)=\sum_{r=-\infty}^{\infty} a_{r}(0) e^{j r \Omega t}, \quad a_{-r}=a_{\tau}^{*},
$$

we find the general solution to be of the form

$$
\begin{aligned}
S(z, t)= & \sum_{r=-\infty}^{\infty} a_{r}(0)\left[\frac{A+j(r \Omega-\delta \omega) \tau_{d 0}}{A e^{-2 a z}+j(r \Omega-\delta \omega) \tau_{d 0}}\right]^{-j(\Gamma B / 2 \alpha A)} \\
& \times e^{-\alpha z} e^{j r \Omega t} .
\end{aligned}
$$




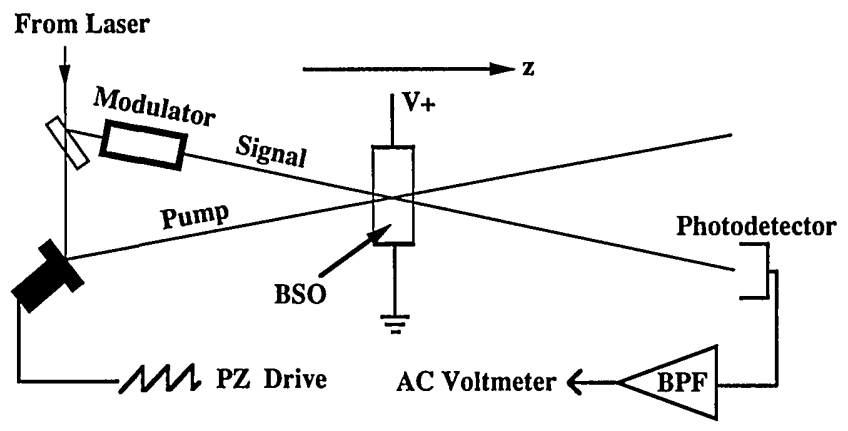

Fig. 1. Experimental arrangement. BPF denotes the bandpass filter; $\mathrm{PZ}$ is piezoelectric.

Let us now consider the special case of square-wave modulation at an angular frequency $\Omega$. The first harmonic of the intensity $I(z, t)=S(z, t) * S(z, t)$ (an observable quantity) may be obtained as

$$
\begin{aligned}
I(z, t)_{\Omega}= & 2 a_{0}(0) a_{1}(0) \\
& \times \operatorname{Re}\left(\left[\left(\frac{A-j \delta \omega \tau_{d 0}}{A e^{-2 \alpha z}-j \delta \omega \tau_{d 0}}\right)^{-j(\Gamma B / 2 \alpha A)} e^{-\alpha z}\right]^{*}\right. \\
& \times\left\{\left[\frac{A-j(\delta \omega-\Omega) \tau_{d 0}}{A e^{-2 \alpha z}-j(\delta \omega-\Omega) \tau_{d 0}}\right]^{-j(\Gamma B / 2 \alpha A)} e^{-\alpha z} e^{j \Omega t}\right. \\
& \left.\left.+\left[\frac{A-j(\delta \omega+\Omega) \tau_{d 0}}{A e^{-2 \alpha z}-j(\delta \omega+\Omega) \tau_{d 0}}\right]^{-j(\Gamma B / 2 \alpha A)} e^{-\alpha z} e^{-j \Omega t}\right\}\right),
\end{aligned}
$$

where Re means the real part and * denotes the complex conjugate. For a square wave $a_{0}(0)$ and $a_{1}(0)$ are in the ratio $1: 4 / \pi$. This expression has three maxima, which occur either when the gain of the dc Fourier component of the amplitude of the signal is optimized or when the gain of the Fourier components at frequencies $\Omega$ and $-\Omega$ are optimized. When the absorption is negligible, these conditions are satisfied for detuning frequencies of $\delta \omega=$ $\delta \omega_{\text {opt }}$ and $\delta \omega=\left(\delta \omega_{\text {opt }} \pm \Omega\right)$, respectively. This is illustrated by Fig. 2(a), which shows a plot of ac gain $\left[I(z, t)_{\Omega} /\right.$ $2 a_{0} a_{1}$ ] against $\delta \omega$ with $\Omega=2 \pi \times 20 \mathrm{rad} / \mathrm{sec}$, a wavelength of $514 \mathrm{~nm}$, a grating spacing of $20 \mu \mathrm{m}, z=1 \mathrm{~cm}$, an incident power density of $5 \mathrm{~mW} / \mathrm{cm}^{2}$, and the material parameters set to typical values for a BSO crystal. ${ }^{6}$

By optimizing the gain of the dc Fourier component, it is possible to obtain ac gain regardless of the modulation frequency. For example, Fig. 3 shows plots of ac gain at $\Omega$ and $3 \Omega$ versus detuning $\delta \omega$, again with negligible absorption and with other parameters as quoted above. Both curves can be seen to have coincident maxima at a detuning frequency of $\delta \omega_{\text {opt }}$. The ac gain reported by Hamel de Montchenault and co-workers ${ }^{1,2}$ was obtained in this fashion.

One further consequence of Eq. (10) is that with no detuning applied to the pump beam, i.e., $\delta \omega=0$, the ac gain may be optimized by setting the angular modulation frequency $\Omega$ equal to $\pm \delta \omega_{\text {opt }}$. This can be seen from Fig. 4(a), which shows a plot of $I(z, t)_{\Omega}$ versus $\Omega$ when $\delta \omega=0$, the other parameters being as above.

It follows from Eq. (10) that the dc gain, obtained by setting $\Omega=0$, is optimized at the same value of detuning as the ac gain obtained when $\Omega \gg \delta \omega$. Further, the optimum dc gain is equal to the square of the optimum ac

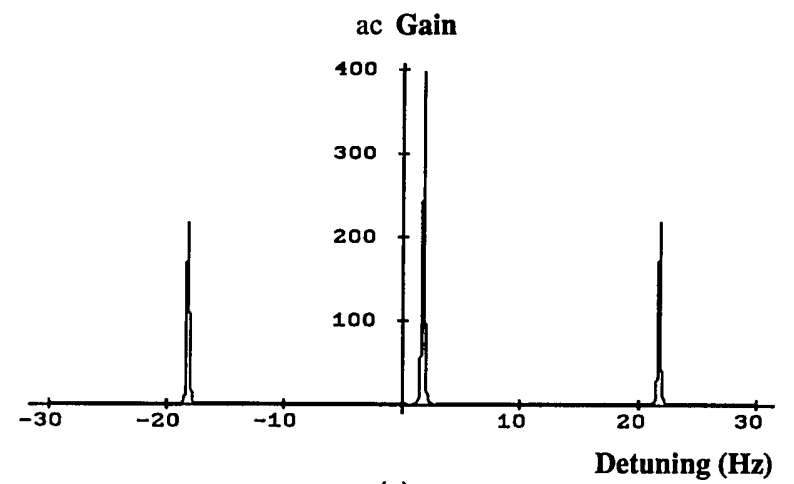

(a)

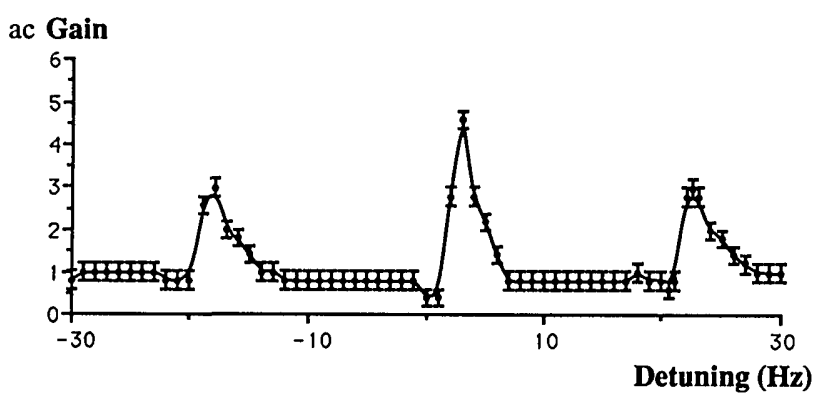

(b)

Fig. 2. (a) Theoretical plot of ac gain against detuning frequency for a signal-beam amplitude modulated by a square wave at $20 \mathrm{~Hz}$. Filter bandpass set to $20 \mathrm{~Hz}$. (b) Experimental results for same arrangement.
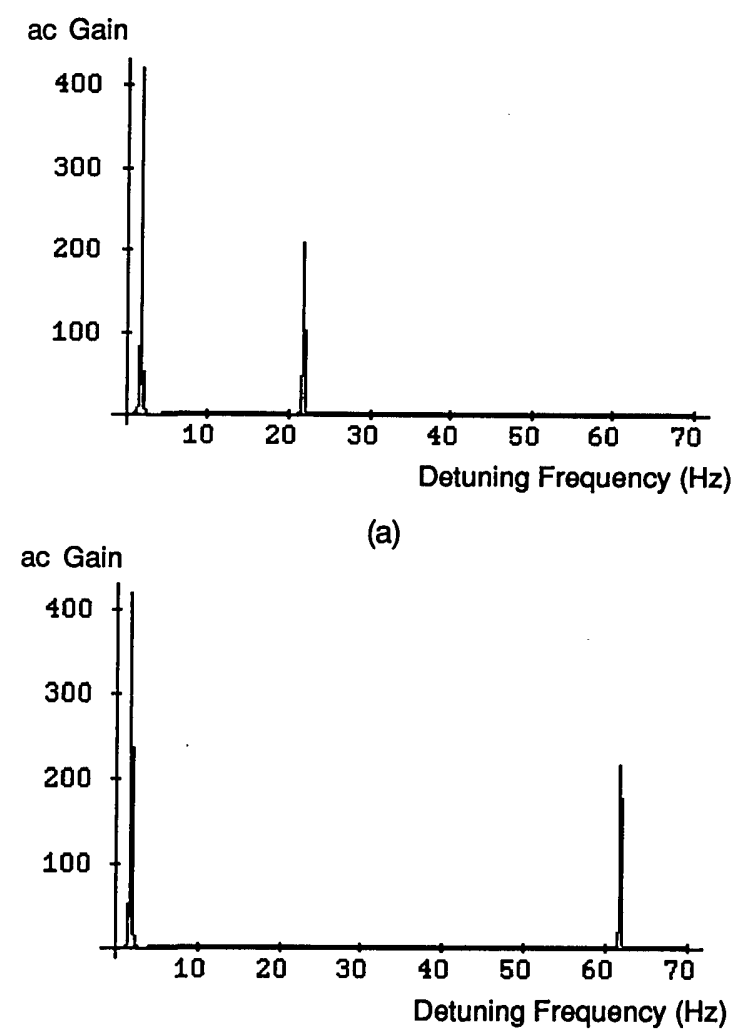

(b)

Fig. 3. Theoretical results for ac gain as a function of detuning frequency for a signal-beam amplitude modulated by a square wave at $20 \mathrm{~Hz}$. (a) Filter bandpass set to $20 \mathrm{~Hz}$, (b) filter bandpass set to $60 \mathrm{~Hz}$. 


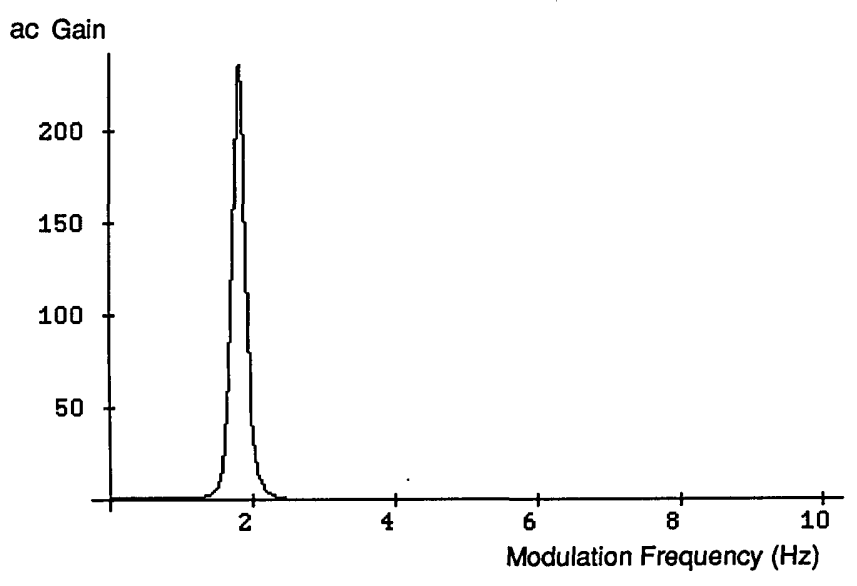

(a)

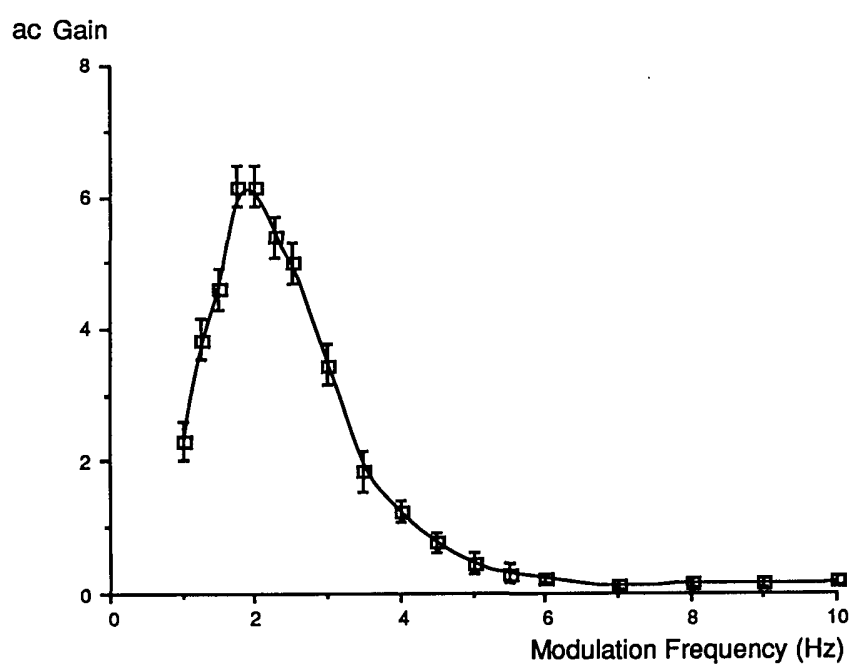

(b)

Fig. 4. Results for ac gain as a function of modulation frequency, with no detuning and filter bandpass set to modulation frequency. (a) Theoretical, (b) experimental.

gain. Experimental results obtained at Thomson-CSF have suggested that this is not the case. ${ }^{7}$ They found that, in practice, the peak value of the square of the ac gain was less than the peak value of the dc gain. In addition, the detuning frequency needed to optimize the dc gain was less than that needed to optimize the ac gain. We suggest that the presence of absorption is responsible for this discrepancy.

In the absence of absorption, through adjustment of the detuning, the gain may be optimized at all points throughout the crystal. When absorption is present, the optimum detuning frequency will vary with distance into the crystal, since $\tau_{d}$ is inversely proportional to the total intensity, which falls off exponentially with distance. From this argument one might expect that absorption would qualitatively result in a broadening of the detuning curve toward lower detuning frequencies.

This is found to be the case when realistic values of the absorption constant are included in Eq. (10). However, the curves for dc and ac gain are affected differently. For the case of ac gain under the conditions $\Omega \gg \delta \omega$ and $\delta \omega \sim \delta \omega_{\text {opt }}$, Eq. (10) simplifies to

$$
\begin{aligned}
I(z, t)_{\Omega} & =4 a_{0}(0) a_{1}(0) \\
& \times \operatorname{Re}\left[\left(\frac{A-j \delta \omega \tau_{d 0}}{A e^{-2 \alpha z}-j \delta \omega \tau_{d 0}}\right)^{-j(\Gamma B / 2 \alpha A)}\right] e^{-2 \alpha z} \cos \Omega t .
\end{aligned}
$$

For dc gain we have

$$
I(z, t)_{\Omega=0}=4 a_{0}(0) a_{1}(0)\left|\left(\frac{A-j \delta \omega \tau_{d 0}}{A e^{-2 \alpha z}-j \delta \omega \tau_{d 0}}\right)^{-j(\Gamma B / 2 \alpha A)}\right|^{2} e^{-2 \alpha z} .
$$

Figure 5 shows plots of the square of the ac gain and the dc gain against detuning for several different values of $\alpha$. (The nonzero values are those measured experimentally at the source wavelengths used in obtaining the experimental results described below.) It is clearly seen that, as the absorption increases, the detuning needed to obtain the optimum value of ac gain decreases less than the detuning needed to optimize dc gain. In addition, the
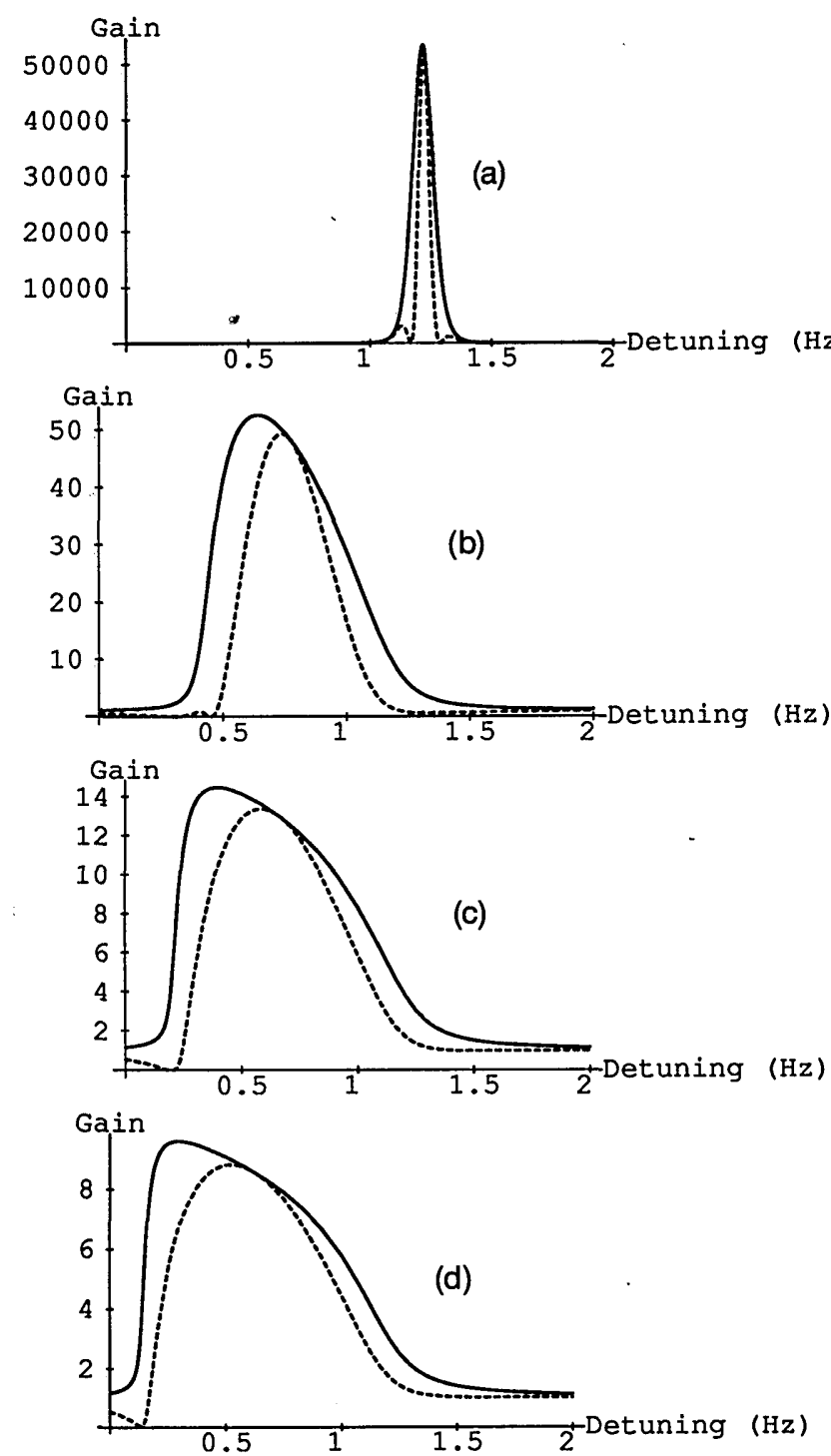

Fig. 5. Theoretical plots of dc gain (solid curves) and (ac gain) ${ }^{2}$ (dashed curves) as a function of detuning frequency for various values of the absorption constant $\alpha$. The nonzero values are those occurring at the source wavelengths that were used to obtain the experimental results in Fig. 7. (a) $\alpha=0$, (b) $\alpha=$ $0.56 \mathrm{~cm}^{-1}$, (c) $\alpha=0.90 \mathrm{~cm}^{-1}$, (d) $\alpha=1.09 \mathrm{~cm}^{-1}$. 
square of the optimum ac gain falls more rapidly than the optimum dc gain. Both these tendencies are in agreement with the observations made at Thomson-CSF. It is worth noting from Eq. (11) that the optimum value of ac gain is obtained at a detuning frequency that is independent of $\Omega$ itself. Furthermore, this detuning frequency is also independent of the precise form of the applied modulation.

\section{EXPERIMENT}

Experimental confirmation of the theory described above was sought by using the arrangement shown in Fig. 1 above. Expanded and collimated beams of light from the 514-nm line of an $\mathrm{Ar}^{+}$laser were used to illuminate the $1 \overline{10}$ face of a 1-cm long crystal of BSO. An electric field of $9 \mathrm{kV} / \mathrm{cm}$ was applied perpendicular to the 110 face, and the light was polarized perpendicular to the 001 face. The pump beam had an intensity of $5 \mathrm{~mW} / \mathrm{cm}^{2}$, and the beam ratio was approximately 400:1. The modulation was provided by a chopper. A bandpass filter was used to obtain the ac output from the photodetector at the fundamental frequency of the square wave.

The modulation frequency was set to $20 \mathrm{~Hz}$, and for a range of different detuning frequencies the ac signal

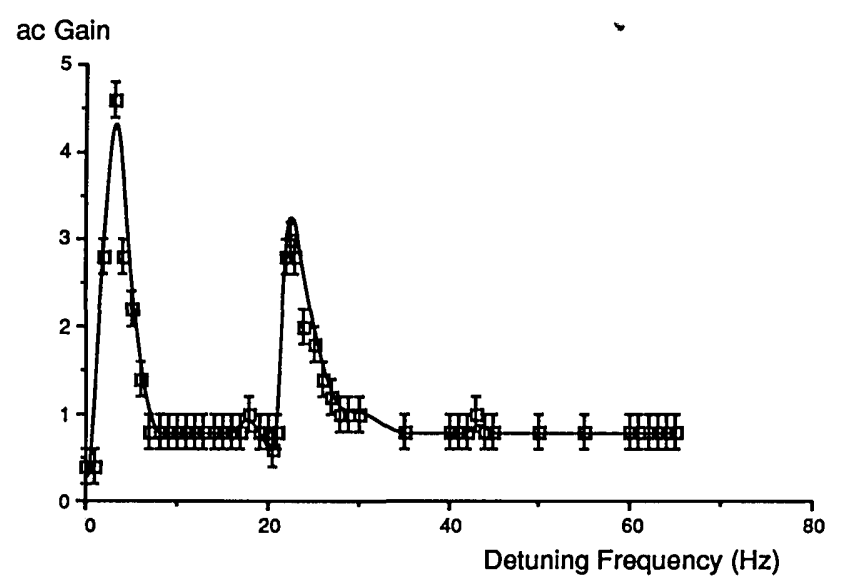

(a)

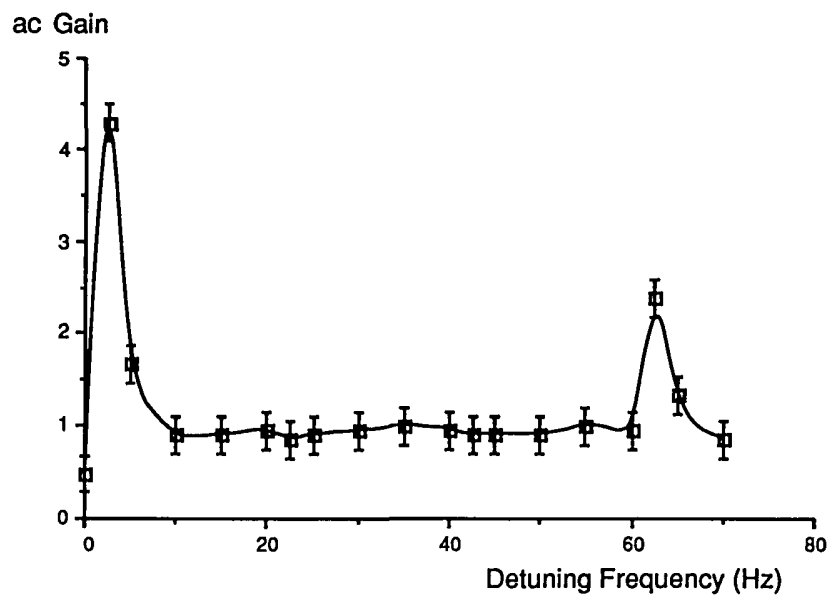

(b)

Fig. 6. Experimental results corresponding to the conditions described in Fig. 3.
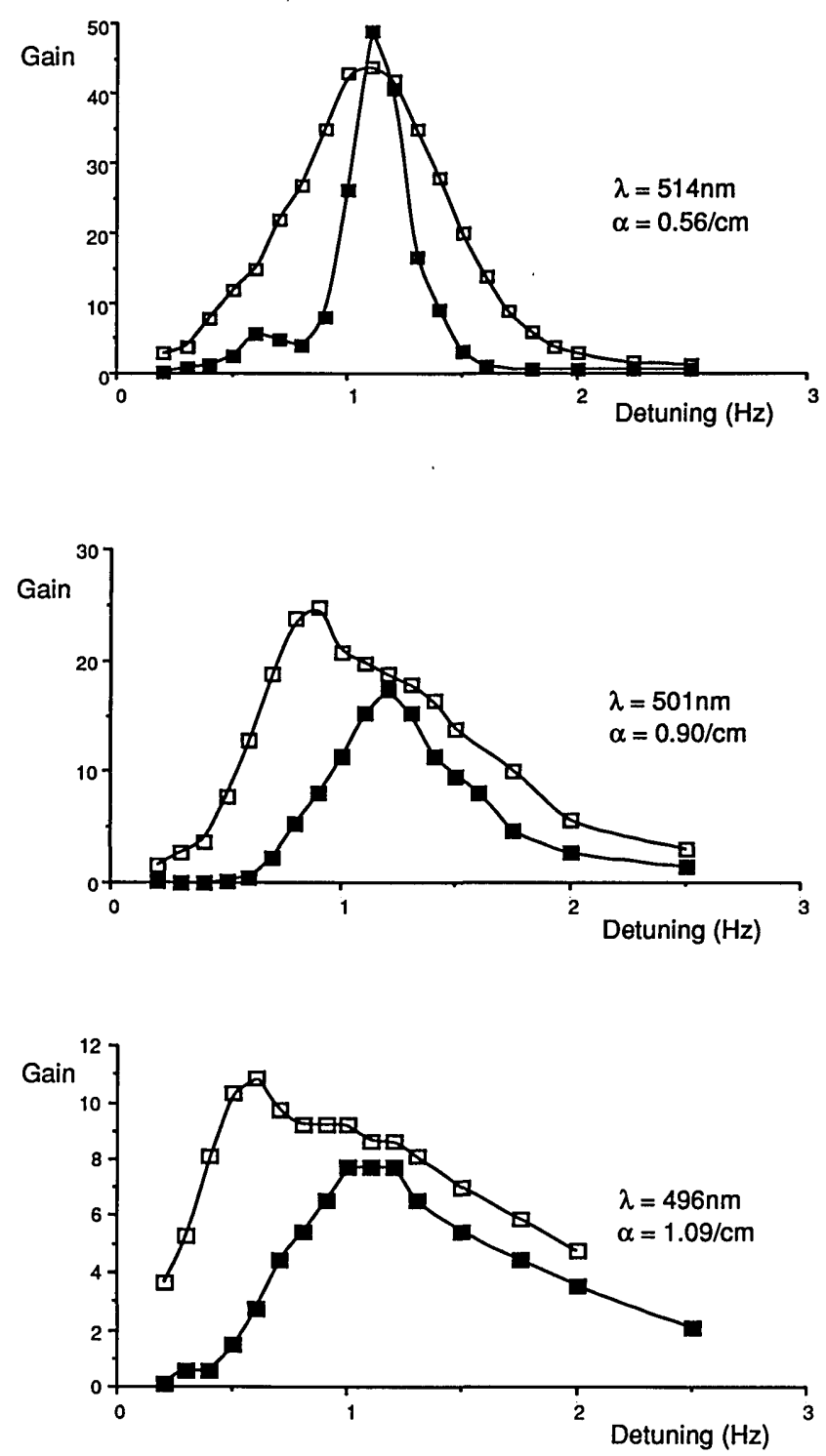

Fig. 7. Experimental values of dc gain (open squares) and (ac gain) ${ }^{2}$ (filled squares) plotted as a function of detuning frequency for three different source wavelengths. Absorption constants were determined at these wavelengths and are indicated in the figure.

amplitude was recorded. These results are shown in Fig. 2(b) above and may be compared with the theoretical plot. The filter bandpass was then centered on $60 \mathrm{~Hz}$, and another set of data was obtained. Detuning curves for the 20- and 60-Hz settings are shown in Fig. 6 and may be compared with the theoretical results shown in Fig. 3 above.

An attempt was also made to duplicate the results shown in Fig. 4(a), for zero detuning. For this experiment the ac gain was measured as a function of the modulation frequency of the signal beam. For each measurement the filter bandpass was centered on the current modulation frequency. The experimental results of (ac gain) $\Omega$ versus $\Omega$ are shown in Fig. 4(b).

The effects of absorption on the detuning curves for dc and ac gain were investigated by obtaining data at 496 , 501 , and $514 \mathrm{~nm}$, at which wavelengths $\alpha$ was measured to be $1.09,0.90$, and $0.56 \mathrm{~cm}^{-1}$, respectively. These values 
were used for the three theoretical plots shown in Figs. 5(b)-5(d). The experimental results are illustrated in Fig. 7, which shows plots of dc gain and (ac gain) ${ }^{2}$ obtained at these wavelengths. The experimental conditions were as described above except that the field was $8 \mathrm{kV} / \mathrm{cm}$ and the total incident intensity was $\sim 2 \mathrm{~mW} / \mathrm{cm}^{2}$ in all cases. For the ac gain measurements a modulation frequency of $220 \mathrm{~Hz}$ was used.

The final experiment confirms the theoretical predictions of the effects of absorption on the detuning curves. Complete agreement between experiment and theory is not to be expected, partly because many of the material parameters used in the simulations were typical values and not necessarily representative of our sample and partly because the effects of optical activity were not taken into account in this study. Given the effects of absorption, the other experimental results may be seen to be in good qualitative agreement with the theoretical predictions.

\section{CONCLUSION}

We have analyzed the amplification of a temporally modulated signal beam by a strong pump beam in a photorefractive medium. It should be stressed that, while we dwelt on the amplification of amplitude-modulated signals, both frequency and phase modulation are also covered by the theory. It is necessary only to compute the frequency spectrum of the signal at the input to the crys- tal in order to obtain the frequency spectrum at any point within the crystal. The theory was found to be in good qualitative agreement with results obtained experimentally using a BSO crystal.

\section{REFERENCES}

1. G. Hamel de Montchenault, B. Loiseaux, and J. P. Huignard, "Amplification of high bandwidth signals through two-wave mixing in photorefractive $\mathrm{Bi}_{12} \mathrm{SiO}_{20}$ crystals," Appl. Phys. Lett. 50, 1794-1796 (1987).

2. G. Hamel de Montchenault and J. P. Huignard, "Two-wave mixing with time-modulated signal in $\mathrm{Bi}_{12} \mathrm{SiO}_{20}$ theory and application to homodyne wavefront detection," J. Appl. Phys. 63, 624-627 (1988).

3. N. V. Kukhtarev, V. B. Markov, S. G. Odulov, M. S. Soskin, and V. L. Vinetskii, "Holographic storage in electrooptic crystals. I. Steady state," Ferroelectrics 22, 949-959 (1979).

4. N. V. Kukhtarev, V. B. Markov, S. G. Odulov, M. S. Soskin, and V. L. Vinetskii, "Holographic storage in electrooptic crystals. II. Beam coupling-light amplification," Ferroelectrics 22, 961-964 (1979).

5. Ph. Refregier, L. Solymar, H. Rajbenbach, and J. P. Huignard, "Two-beam coupling in photorefractive $\mathrm{Bi}_{12} \mathrm{SiO}_{20}$ crystals with moving grating: theory and experiments," J. Appl. Phys. 58, 45-57 (1985).

6. R. A. Mullen and R. W. Hellwarth, "Optical measurement of the photorefractive parameters of $\mathrm{Bi}_{12} \mathrm{SiO}_{20}$," J. Appl. Phys. 58, 40-44 (1985).

7. J. P. Huignard and B. Loiseaux, Thomson-CSF, Laboratoire Central de Recherches, Domaine de Corbeville, 91404 Orsay Cedex, France (personal communication, September 1989). 